
\title{
25 Research Soure \\ Hierarchical Porous Aero-Cryogels for Wind Energy Enhanced Solar Vapor Generation
}

\section{Shuai Li}

Guilin University of Technology

Xiaochun Yang

Guilin University of Technology

\section{Yingying $\mathrm{He}$}

Guilin University of Technology

Yanan Wang

Guilin University of Technology

Daogui Liao

Guilin University of Technology

\section{Yunhua Chen}

South China University of Technology

Huihong Xie

Guilin University of Technology

Hongxia Liu ( $\square$ hliu28551@gmail.com )

Guilin University of Technology https://orcid.org/0000-0003-1698-0720

\section{Li Zhou}

Guilin University of Technology

\section{Research Article}

Keywords: Aero-cryogel, Hierarchical porous structure, Solar vapor generation, Water purification

Posted Date: August 20th, 2021

DOl: https://doi.org/10.21203/rs.3.rs-792434/v1

License: (c) (i) This work is licensed under a Creative Commons Attribution 4.0 International License.

Read Full License

Version of Record: A version of this preprint was published at Cellulose on November 25th, 2021. See the published version at https://doi.org/10.1007/s10570-021-04335-2. 


\section{Abstract}

An integrated aero-cryogel (A-CG) monolith with hierarchical porous structure was developed by intercrosslinking of cellulose nanofiber (CNF)/polylactic acid (PLA) nanocomposite aerogel and carboxymethyl cellulose (CMC) cryogel. The photothermal nanoparticles-enriched $\mathrm{CMC}$ cryogel phase served as a sunlight absorbing layer, exhibiting a broadband sunlight absorption of $98 \%$. Due to the large amount of weakly bounded water molecules, the swelled CMC cryogel possessed a lower evaporation enthalpy than that of pure water, which facilitates water evaporation, while the nanocomposite aerogel phase acted as an excellent thermal insulator and afforded highly efficient water transport channels. Thus, the developed A-CG monolith supported by insulated polystyrene foam to protrude above the water surface, could reach an evaporation rate of $2.16 \mathrm{~kg} \mathrm{~m}^{-2} \mathrm{~h}^{-1}$ under an irradiation of 1 Sun $\left(100 \mathrm{mw} / \mathrm{cm}^{2}\right)$ with an efficiency of $93.6 \%$. More remarkably, when the wind energy was imparted, an evaporation rate of $5.67 \mathrm{~kg} \mathrm{~m}^{-2} \mathrm{~h}^{-1}$ was achieved at a wind speed of $3 \mathrm{~m} \mathrm{~s}^{-1}$. The high-efficiency purification outcomes of various raw water demonstrate the great potentials of A-CG material in solar vapor generation.

\section{Introduction}

The sun provides a staggering amount of energy to earth, with an annual solar flux of approximately $1.2 \times 10^{5}$ terawatt, which far exceeds the human needs in today's society (Crabtree \& Lewis, 2007; Yu, Yan, Ullah, Chen, \& Li, 2020). Therefore, the effective collection and utilization of solar energy can effectively alleviate the increasingly severe shortage of fossil fuel resources. Thus far, solar energy has been converted into electricity, heat, and chemical energy for use in various applications (X. Zhou, Guo, Zhao, Shi, \& Yu, 2020). Among them, solar vapor generation is an appealing technology because it uses green and sustainable solar energy to extract fresh water from various types of raw water with minimum environmental impact compared with other water purification technologies, and provides new approach toward solving the problem of water scarcity (X. Li et al., 2019; Liu, Huang, Liu, Hu, \& Zhou, 2019). In recent years, a higher conversion efficiency (approximately 90\%) of solar vapor generation has been achieved by a floating porous structure as the solar vapor generator (SVG) on the water surface to achieve thermal localization (Ni et al., 2016; Panpan Zhang et al., 2019). Various photothermal materials with high solar absorption, such as plasmonic metal and metal oxide nanoparticles (Guo et al., 2019; Xu et al., 2019; Yuan et al., 2020) carbon-based materials (N. Li et al., 2020; L. Wang et al., 2020; Wu et al., 2019; P. Zhang, Li, Lv, Zhao, \& Qu, 2017) and polymers (S. Li, Y. He, et al., 2020; Li, Li, Bertelsmann, \& Fan, 2019; Zou et al., 2020) have been shown to be capable of collecting approximately all energy in the solar spectrum and converting it into heat.

Hydrogel is a three-dimensional cross-linked polymer network swollen with water (X. Zhou, Guo, Zhao, \& Yu, 2019). The water-solubilizing groups in the polymer chain (such as hydroxyl, carboxylic acid, and sulfonic acid groups) can interact with water molecules through hydrogen bonds and electrostatic interaction, and thereby reduce the energy required for vaporization (X. Zhou, Guo, et al., 2019; X. Zhou, Zhao, Guo, Rosenberger, \& Yu, 2019). Undergoing a freeze-drying and re-hydration process, hydrogel can be transferred into cryogel with macroporous structure. When the swelled cryogel is utilized to build SVG, 
a record evaporation rate of $3.6 \mathrm{~kg} \mathrm{~m}^{-2} \mathrm{~h}^{-1}$ with an efficiency of $92 \%$ can be achieved under 1 Sun (X. Zhou, Zhao, et al., 2019).

However, a single cryogel structure tends to have a density close to $1 \mathrm{~g} \mathrm{~cm}^{-3}$ after being completely swollen, and can only be suspended below the water surface; therefore, its actual evaporation area (the surface area of the SVG in bare air) is very small. Moreover, the higher water content in the cryogel tends to result in the high thermal conductivity of the SVG to decentralize the heat and reduce the overall efficiency (X. Zhou et al., 2020). Surprisingly, a cylindrical photothermal reservoir, which required neither immersion in nor contact with bulk water, was used to effectively increase the evaporation area and reduce heat loss, thereby a steam generation rate of $4.0 \mathrm{~kg} \mathrm{~m}^{-2} \mathrm{~h}^{-1}$ was achieved (Wu et al., 2019). However, the limitation of continuous water supply could cause the structural defects of the reservoir due to dehydration during the water evaporation process. Additionally, the SVG is often used in open areas, which often have abundant wind energy resources. Although various studies have reported the improvement of SVG evaporation performance after wind energy input (J. Li et al., 2020; N. Li et al., 2020), the influence of wind (wind direction, water vapor temperature, etc) on the evaporation process still requires further investigation.

In this work, an integrative aero-cryogel (A-CG) SVG with hierarchical porous structure was constructed by combining carboxymethyl cellulose (CMC) hydrogel with a special cellulose nanofiber (CNF)/polylactic acid (PLA) composite aerogel through a simple cross-linking and freeze-drying process. The photothermal nanoparticles-enriched $\mathrm{CMC}$ cryogel with macroporous structure containing photothermal nanoparticles as a sunlight absorbing layer exhibited a broadband sunlight absorption of $98 \%$. Due to that the hydration of hydroxyl on the CMC chains generates a large amount of weakly bounded water molecules, the swelled CMC cryogel possessed a lower evaporation enthalpy $\left(1.70 \mathrm{~kJ} \mathrm{~g}^{-1}\right)$ than that of pure water $\left(2.66 \mathrm{~kJ} \mathrm{~g}^{-1}\right)$. While the ultra-hydrophilic, high porous and low thermal conductive composite aerogel acted as an excellent thermal insulator and afforded highly efficient water transport channels. Thus, the ingeniously designed A-CG/F, an optimal solar vapor generator in which the A-CG was supported by insulated polystyrene foam to protrude above the water surface, could reach an evaporation rate of $2.16 \mathrm{~kg} \mathrm{~m}^{-2} \mathrm{~h}^{-1}$ under an irradiation of 1 Sun $\left(100 \mathrm{mw} / \mathrm{cm}^{2}\right)$ with an efficiency of $93.6 \%$. Moreover, the different evaporation characteristics of SVG at different positions (light-absorbing side, windward side, leeward side, and so on) were also discussed, and the promotion effect of wind on the evaporation process was further elucidated. Wind energy exerted an obvious promotion effect on the evaporation rate of SVG, and thus achieves a high evaporation rate of $5.67 \mathrm{~kg} \mathrm{~m}^{-2} \mathrm{~h}^{-1}$ at a wind speed of $3 \mathrm{~m} \mathrm{~s}^{-1}$. This is attributed to the fact that the wind energy promotes the absorption of heat from the environment by the SVG to produce water vapor below the ambient temperature. Finally, the A-CG can purify many types of raw water, and has enormous application potential in the fields of seawater desalination, and domestic and industrial wastewater purification.

\section{Experimental Section}




\subsection{Materials}

The 1, 2-dichloroethane (DCE), hydrochloric acid, glutaraldehyde (GA), ammonium persulfate (APS), aniline, CMC (600-1000 mpa.s), Methylene blue, rhodamine B (RhB), and ion standard solution were purchased from Aladdin Chemistry Co. Ltd. (Shanghai, China) and used directly. Polylactic acid (PLA) was purchased from the Chi Mei Industrial Factory (Taiwan). The aqueous solution of the CNFs was purchased from Qihong Technology Co., Ltd (Guilin, China).

\subsection{Preparation of cross-linked CNF/PLA composite aerogel}

First, $0.5 \mathrm{ml}$ of glutaraldehyde were added to $0.5 \mathrm{wt} \% \mathrm{CNF}$ aqueous dispersion $(200 \mathrm{ml})$ and stirred overnight. Then, hydrochloric acid ( $1 \mathrm{M}$ ) was added dropwise to adjust the mixture above the $\mathrm{pH}$ of 4 . The acid mixture as the water phase and the 1, 2-dichloroethane (containing $2 \mathrm{wt} \% \mathrm{PLA}$ ) as the oil phase with a water-oil volume ratio of 4:1 were emulsified using a sonicator at $400 \mathrm{~W}$ for $5 \mathrm{~min}$. After repeating the ultrasonication treatment five times, a CNF-stabilized Pickering emulsion gel was formed. Subsequently, a certain volume of gel was transferred to a particular mold, froze in the refrigerator for 12 hours, and then freeze-dried $\left(-49^{\circ} \mathrm{C}, 20 \mathrm{~Pa}\right)$ for $48 \mathrm{~h}$ to obtain a cross-linked CNF/PLA composite aerogel with a specific shape.

\subsection{Preparation of PANI nanoparticles}

In a typical synthesis, $36.34 \mathrm{~g}$ of APS, $16.7 \mathrm{ml}$ of hydrochloric acid (12 M), and $177.6 \mathrm{ml}$ of Distilled water (DI) water were evenly mixed by stirring. Then, $5.8 \mathrm{ml}$ of aniline solution were added to polymerize in an ice water bath in the dark. After 6 hours, a PANI suspension was obtained. Repeated centrifugation and water washing treatment were subsequently conducted for several times. Finally, a certain concentration of PANI aqueous dispersion was saved for subsequent use.

\subsection{Preparation of cryogels and aero-cryogels}

A certain amount of PANI aqueous dispersion ( $5 \mathrm{wt} \%)$, DI water, and glutaraldehyde (Table S1 for details) were added to $12 \mathrm{ml}$ of $\mathrm{CMC}$ aqueous solution ( $6 \mathrm{wt} \%$ ) and stirred for $8 \mathrm{~h}$. The stirring continued for $5-10$ min after adding hydrochloric acid $(4 \mathrm{M}, 1 \mathrm{ml})$ dropwise into the above-mentioned mixture. Then, the reaction solution was quickly transferred to a Petri dish for a further gelation reaction at room temperature for $6 \mathrm{~h}$. Repeated freezing and thawing treatment was carried out approximately five times to enhance the gelation. Finally, a cryogel was obtained by freeze-drying and rehydrating the wet crosslinked products for $48 \mathrm{~h}$. The resulting cryogels are referred to as CG-X, where $\mathrm{X}$ represents the different PANI contents in the cryogel, which are listed in Table S1.

The procedure of preparing A-CGs is similar to that of CGs, except for the fact that CNF/PLA composite aerogel with different shapes was placed into the viscous aqueous reaction solution during the gelation reaction. The resulting A-CGs are referred to as A-CG-X, where $X$ represents the type of cryogel used. Additionally, the mushroom-shaped A-CGs with PS foams are referred to as A-CG/F.

\subsection{Solar vapor generation experiment}


Steam generation experiments were conducted at the room temperature of $25^{\circ} \mathrm{C}$ and humidity of $55 \%$ using a solar simulator (CEL-S500, Beijing Zhongjiao Jinyuan Technology Co., Ltd.) with an optical filter for the standard AM 1.5G spectrum. The solar flux was calibrated using a full spectrum glare optical power meter (CEL-NP2000, Beijing Zhongjiao Jinyuan Technology Co., Ltd.). The mass change of the 3.5 $w t \% \mathrm{NaCl}$ solution was expressed and recorded by the change of the electronic balance (FA2004) during the experiment over 2 hours. The wind speed was calibrated using a high-precision wind speed measuring instrument (AS856, SMART SENSOR).

\subsection{Characterization}

The morphology and structure of the samples were characterized using field emission scanning electron microscopy (FE-SEM; Hitachi S-4800, Japan) for which each sample was gold-sputtered. The Fourier transform infrared (FT-IR) sample spectra were measured using a Nicolet NEXUS 470 infra-red spectrometer (USA) operating in transmission mode for wavenumbers ranging from 400 to $4000 \mathrm{~cm}^{-1}$ (resolution: $2 \mathrm{~cm}^{-1}$ ). The evaporation and melting behavior of water in CG was observed by differential scanning calorimeter (TA Q2000, USA). The mechanical properties of the aerogels were obtained by compression testing using the Shimadzu AG-201 universal material testing machine with a step length of $120 \mathrm{~mm} / \mathrm{min}$, compression height of $10 \mathrm{~mm}$, and load of $5 \mathrm{kN}$. The light reflection and transmission in the wavelength range of $300 \mathrm{~nm}$ to $2500 \mathrm{~nm}$ for SVGs were measured using an UV-vis-NIR spectrophotometer (Jasco V-570, Japan) coupled with the integrating sphere test. Infrared (IR) photographs were captured using an IR camera (Fluke, Ti32, USA). The ionic concentrations were measured using an inductively coupled plasma optical emission spectrometer (PerkinElmer, Optima 8000, USA). The absorption spectra of Methylene blue and RhB water were measured using a UV-vis spectrometer (PerkinElmer, Lambda 750S, USA), and digital photos were captured using a commercial camera (Canon, IXUS 125HS, Japan).

\section{Results And Discussion}

The schematic diagram for the construction of SVGs in our strategy is shown in Fig. 1. Owing to the unique interaction between the polymer chains and the water in the swelled cryogel, the cryogel was first considered as an ideal SVG. However, owing to the high density of the swelled cryogels, it is difficult to float them on water for a long period of time (Fig. S1). Therefore, a crosslinked CNF/PLA composite aerogel based on Pickering emulsion technology was proposed as the cryogel substrate owing to its advantages: the CNF/PLA composite aerogels can provide higher water resistance and anti-compression properties, while still maintaining similar porosity, thermal conductivity, and hydrophilicity as the pure CNF aerogel (Fig. S2, S3) (S. Li, Y. He, et al., 2020; S. Li, C. Zhou, et al., 2020). Additionally, their specific shapes can be obtained through an ingeniously designed mold (Fig. S4).

As an important derivative of cellulose, $\mathrm{CMC}$ is one of the most widely used eco-friendly materials with a rich content of carboxyl and hydroxyl groups on the cellulose backbone, and is also available in large amounts (Shu et al., 2020). Thus, in this study, CMC was considered as an ideal candidate for preparing 
cryogels. As shown in Fig. 1a, the CMC cryogel containing polyaniline (PANI) nanoparticles were prepared by a simple one-pot in situ crosslinking reaction through glutaraldehyde (GA). The detailed preparation process and formulations for the cryogels (CG) are presented in Sect. 2.4 and Table S1, respectively, with detailed abbreviations. The chemical composition of the CGs was verified by the Fourier transform infrared (FTIR) spectrum shown in Fig. 1b. In the CMC spectrum, the peaks at $3414 \mathrm{~cm}^{-1}, 1617 \mathrm{~cm}^{-1}$, $1054 \mathrm{~cm}^{-1}$, and $1420 \mathrm{~cm}^{-1}$ represent the stretching vibrations of $\mathrm{O}-\mathrm{H},-\mathrm{COOH}$, and $\mathrm{C}-\mathrm{O}-\mathrm{C}$, and the scissoring vibration of $-\mathrm{CH}_{2}$, respectively. In the $\mathrm{CG}-0$ spectrum, a new absorption peak corresponding to the aldehyde peak $(\mathrm{CHO})$ appeared at $1716 \mathrm{~cm}^{-1}$, which confirms the formation of a crosslinked network (Fig. 1C). For the PANI spectrum, the characteristic absorption peaks at $1561 \mathrm{~cm}^{-1}, 1480 \mathrm{~cm}^{-1}, 1301 \mathrm{~cm}^{-}$ $1,1246 \mathrm{~cm}^{-1}$, and $1137 \mathrm{~cm}^{-1}$ correspond to the $\mathrm{C}=\mathrm{C}$ stretching deformation of the quinoid rings, $\mathrm{C}=\mathrm{C}$ stretching deformation of the benzenoid rings, $\mathrm{C}-\mathrm{N}$ stretching vibration of the aromatic amine, doped $\mathrm{C}$ $\mathrm{N} .{ }^{+}$aromatic amine peak, and in-plane bending vibration of the plane deformation of the $\mathrm{C}-\mathrm{H}$ in the benzene ring, respectively. The characteristic peaks of $\mathrm{CMC}, \mathrm{PANI}$, and GA are clearly observed in the spectrum of CG-5, which confirms the formation of CMC cryogel crosslinked by GA and the successful introduction of PANI in CG-5.

When the CNF/PLA composite aerogel with a specific shape was introduced into the CMC viscous aqueous solution containing crosslinkers, the viscous solution was infiltrated into the bottom of the aerogel by the capillary force. Accompanied by the subsequent cross-linking and gelation reaction, the aerogel and hydrogels were firmly combined through chemical bonds and hydrogen bonds. The crosslinking interaction was further enhanced by repeated freezing and thawing treatment, and an integrated A-CG was finally prepared through the freeze-drying and rehydrating process (Fig. 1a). The resultant A-CG exhibited excellent interface bonding, as can be initially inferred from the phenomenon shown in Fig. S5. When the cylindrical aerogel (diameter of $1.2 \mathrm{~cm}$ ) was held and lifted, the cryogel after being completely swollen with water and Petri dish (total weight of approximately $35 \mathrm{~g}$ ) were also lifted, and the A-CG still maintained a complete structure. To further evaluate the robustness and stability of the A-CG and interface bonding, the A-CG with a particular shape shown in Fig. $\mathbf{S 6}$ was continuously subjected to a series of severe conditions, such as ultrasonic agitation ( $400 \mathrm{~W}, 8 \mathrm{~h})$, boiling water $\left(98^{\circ} \mathrm{C}, 4 \mathrm{~h}\right)$, strong oxidizing acid $\left(1 \mathrm{M} \mathrm{H}_{2} \mathrm{SO}_{4}, 24 \mathrm{~h}\right)$ environments, and even soaking in water for 30 days. After these rigorous tests, the appearance of A-CG did not significantly change, and the A-CG still exhibited an excellent self-floating property, which again confirms the structural stability and strong interface bonding force of the A-CG.

Additionally, the morphologies of the vertical section of the A-CG-5 shown in Fig. 2a, b were also investigated using scanning electron microscopy (SEM). In the A-CG, the cryogel part (CG-5) as the solar absorber of SVG had a typical macroporous structure with a pore diameter of approximately several hundreds microns (Fig. 2c). The PANI photothermal material was randomly distributed onto the pore wall (Fig. 2d). The aerogel part also had a large number of interconnected micron pore structures. Compared with the cryogel, the aerogel had smaller pore diameter, and smoother and flatter pore wall structure (Fig. $2 e, f)$. These relatively small pore structures will have stronger capillary forces to ensure sufficient water 
supply during the evaporation process (Z. Wang, Wu, He, Peng, \& Li, 2021). When the bottom of the aerogel was placed in an aqueous solution saturated with methylene blue, the excellent hydrophilicity and strong capillary force could overcome gravity to draw water to the top of the aerogel, which indicates its excellent water transport ability (Fig. S7). In the cross-sectional SEM image, a hierarchical pore structure between the aerogel and the cryogel can be clearly observed (Fig. $2 \mathrm{~g}$ ). The high magnification SEM micrograph (Fig. 2h) also shows that the aerogels and cryogels were effectively combined, which ensures the structural stability and facilitates continuous water transport.

As has been previously reported, the water in the cryogel after being completely swollen with water can be divided into bound water, intermediate water, and free water (X. Zhou et al., 2020; X. Zhou, Zhao, et al., 2019). Additionally, the evaporation enthalpy of the water in the cryogels is greatly influenced by the intermediate water owing to the weak hydrogen bond interaction between the water and the polymer molecular chains. Hence, differential scanning calorimetry (DSC) was used to reveal the phase change of water in the CGs. In Fig. 3a, the melting point peaks corresponding to the intermediate water (blue dotted curves) and free water (blue curves) are clearly observed, which is consistent with the result reported by Yu et al (X. Zhou et al., 2020; X. Zhou, Zhao, et al., 2019). This confirms that the CMC molecular chains can facilitate water activation transformation from free water to intermediate water owing to the formation of hydrogen bond interaction between the carboxyl group in the $\mathrm{CMC}$ and water, which leads to the lower evaporation enthalpy of CGs compared with pure water. Hence, to accurately calculate the water steam generation efficiency of the final obtained SVG, the precise enthalpy of the evaporation of A-CGs with different PANI content was investigated (see Supplementary Materials Sect. 1.1 for details). The evaporation rate $(\dot{m})$ can be calculated as follows (Y. Wang et al., 2018):

$$
\dot{m}=d_{m} / S d_{t}
$$

where $m$ is the mass of the evaporated water, $S$ is the evaporation area, and $t$ is the evaporation time. The evaporation rate of pure water, A-CG-0, A-CG-2, A-CG-5, and A-CG-8 without solar irradiation was $0.043,0.063,0.0586,0.0582$, and $0.0567 \mathrm{~kg} \mathrm{~m}^{-2} \mathrm{~h}^{-1}$, respectively (Fig. 3b). The evaporation enthalpies of water in all A-CGs were significantly lower than that of water with the same evaporation area. Moreover, with the continuous increase of PANI content, the evaporation enthalpy of water in A-CG-0, A-CG-2, A-CG-5, and A-CG-8 also increased, and was $1.50,1.65,1.66$, and $1.70 \mathrm{~kJ} \mathrm{~g}^{-1}$, respectively. The increase of the evaporation enthalpy is attributed to the fact that the $\mathrm{N}-\mathrm{H}$ in PANI can also form hydrogen bonds with $\mathrm{COOH}$ in $\mathrm{CMC}$, which may weaken the hydrogen bond interaction between the $\mathrm{CMC}$ and water and diminish the formation of intermediate water. Compared with A-CG-8, the A-CG-2 and A-CG-5 had the lower water evaporation enthalpy for accelerating the water evaporation of the final obtained SVG.

Additionally, high solar absorption is an essential prerequisite for an SVG with high performance. Therefore, the effect of the PANI content in the CGs on the light absorption of the CGs was also investigated using an ultraviolet-visible near-infrared (UV-Vis-NIR) spectrophotometer equipped with an integrating sphere. The transmission and reflection spectra of CGs with different PANI content between 
$200 \mathrm{~nm}$ and $2500 \mathrm{~nm}$ were measured to further calculate their absorption spectrum. (Fig. 3c, d) The A-CG0 without PANI exhibited a high diffuse reflection (approximately 40\%) in the visible light region, which indicates lower absorption. After adding the PANI light-absorbing material, the diffuse reflection of A-CG-2 to light was significantly reduced; therefore, high light absorption (approximately $98 \%$ ) was achieved. Notably, the absorption of A-CG-5 and A-CG-8 wasslightly higher than that of A-CG-2. Moreover, the water contact angle test confirms that all CGs with different PANI content have excellent hydrophilicity and wettability (Fig. S8). In conclusion, a higher PANI content can enhance the light absorption of the CG. However, a higher PANI content can also weaken the interaction between the $\mathrm{CMC}$ and the water molecules. Therefore, CG-5 was selected to construct the SVG used in the subsequent experiment.

Apart from the high solar absorption, the thermal management capacity of the SVGs is also as an important factor influencing the acceleration of evaporation. For comparison, three SVG types with different shape were designed and their diagrams are shown in Fig. 4. As stated above, once the CG-5 were put in a breaker full of water, they sank to the bottom of the breaker (Fig. S1). Although the A-CGs can self-float on water, all A-CGs were still located below the water surface (Fig. 4, S6). Hence, the CG and A-CG as SVGs maybe result in more heat loss owing to the high thermal conductivity of water. Thus, we propose a mushroom-shaped evaporator supported by insulated polystyrene (PS) foam (A-CG/F) (Fig. 4). Compared with A-CG, A-CG/F can protrude above the water surface through the buoyancy of the PS foam to achieve a larger evaporation area. Additionally, PS can be used as a heat insulation layer to further limit the heat loss to the lower bulk water.

To further verify this conclusion, the thermal conductivity of the CG-5 and obtained CNF/PLA aerogel was calculated in accordance with the experimental method described in Supplementary Materials Sect. 1.2 and the corresponding results shown in Fig. S9. Obviously, the thermal conductivity of the CNF/PLA aerogel filled with water is $0.305 \mathrm{~W} \mathrm{~m}^{-1} \mathrm{~K}^{-1}$. This is much lower than that of the fully swollen CG-5 $\left(0.570 \mathrm{~W} \mathrm{~m}^{-1} \mathrm{~K}^{-1}\right)$, which is close to that of pure water $\left(0.609 \mathrm{~W} \mathrm{~m}^{-1} \mathrm{~K}^{-1}\right)$ (N. Shalkevich, A. Shalkevich \& Burgi, 2010). Undoubtedly, the higher thermal conductivity accelerated the heat loss to the bulk water and weakened the evaporation rate. An infrared (IR) thermal imaging camera was used to dynamically monitor the temperature changes of pure water, CG, A-CG, and A-CG/F under the irradiation of 1 Sun. Owing to the high thermal conductivity and high light transmittance of water, the temperature of pure water under irradiation increased almost uniformly (Fig. 4). The CG sank to the bottom of the water after being completely swollen, and the sunlight was absorbed by the CG through the water. Hence, the temperature of the water surface and CG was higher than that of other areas, which is consistent with the bottom heating phenomenon proposed by Deng et al (Tao et al., 2018). With the introduction of the CNF/PLA aerogel, on one hand, the A-CG easily floated on the water surface, absorbed solar energy, and converted this energy into heat. On the other hand, a small part of the generated heat was conducted through the aerogel to the bulk water owing to the lower thermal conductivity of the aerogels, which led to the rapid increase of the temperature of the top water surface and the slow increase of the temperature of the bulk water. Which reached up to $35.1^{\circ} \mathrm{C}$ and $21.6^{\circ} \mathrm{C}$, respectively, when the evaporation process was stable. Interestingly, A-CG/F can further reduce the heat loss to the bulk water. After a long period of 
irradiation, much less heat was lost to the bulk water, which caused the temperature of the bulk water to increase only slightly by $0.1^{\circ} \mathrm{C}$. Hence, A-CG/F is considered to be an ideal SVG with excellent thermal management capability.

The vapor evaporation performances of the SVGs under the irradiation of 1 Sun were further evaluated. As shown in Fig. $5 a$ and $b$, the average evaporation rate was $0.46,0.48,1.84$, and $2.16 \mathrm{~kg} \mathrm{~m}^{-2} \mathrm{~h}^{-1}$, corresponding to pure water, CG, A-CG, and A-CG/F, respectively. This high evaporation rate for the A-CG/F is attributed to the following reasons. First, the abundant hydrophilic groups on the CMC surface can reduce the evaporation enthalpy of water. As stated above, the evaporation enthalpy of water in A-CG-5 was $1.66 \mathrm{~kJ} \mathrm{~g}^{-1}$, which is much lower than that of pure water (approximately $2.26 \mathrm{~kJ} \mathrm{~g} \mathrm{~g}^{-1}$ ). However, macroporous cryogels have high light absorption within a broad band. Secondly, the aerogel with excellent hydrophilicity and relatively small pore structure endows the A-CG/F with stronger capillary action for pumping water. Additionally, a larger evaporation area and a minor heat loss to the bulk water were achieved owing to the protrusive structure of the A-CG/Fs through the buoyancy of the PS foam, which can extremely accelerate the evaporation of water. he steam generation efficiency $\left(\eta_{t h}\right)$ is defined as follows:

$$
\eta_{t h}=\dot{m} h_{L V} / C_{o p t} q_{i}
$$

All steam generation efficiencies were calculated by subtracting the evaporation rate without solar irradiation (Fig. 5b). Notably, $C_{\text {opt }}$ is the optical concentration and $q_{i}$ is the standard solar irradiation (1 $\left.\mathrm{kw} / \mathrm{m}^{2}\right)$, which can be calibrated using a full spectrum glare optical power meter. Here, $h_{L V}$ was determined by the actual evaporation enthalpy in the experiment described in Supplementary Materials Section 1.1. Thus, the steam generation efficiency of the SVGs was calculated as $26.1 \%, 31.9 \%, 82.1 \%$, and $93.6 \%$, corresponding to pure water, CG, A-CG, and A-CG/F, respectively (Fig. 5c).

Energy balance analysis was conducted to further demonstrate the excellent performance of A-CG and A$\mathrm{CG} / \mathrm{F}$ in terms of the effective use of heat (see Supplementary Materials Sect. 1.3 for details). As shown in Fig. 5 d, the A-CG device had a radiation loss of $5.5 \%$, convection loss of $4.5 \%$, and conduction loss of $11.4 \%$. Compared with A-CG, the radiation and convection losses of A-CG/F slightly increased to $5.7 \%$ and $5.8 \%$, respectively (Fig. 5e). This is attributed to the increase in the area of heat exchange with the environment owing to the raised A-CG/F structure, which resulted in an increased evaporation area. In contrast, the conduction loss sharply decreased to $0.5 \%$, and this was the primary reason for the PS ring with an extremely low thermal conductivity of $0.03 \mathrm{~W} \mathrm{~m}^{-1} \mathrm{~K}^{-1}$ (T. Li et al., 2018) exhibiting a better thermal insulation effect compared with the water-filled aerogel, whose thermal conductivity was $0.305 \mathrm{~W}$ $\mathrm{m}^{-1} \mathrm{~K}^{-1}$.

A cyclic performance test was also performed on the A-CG/F irradiated under 1 Sun for $2 \mathrm{~h}$ each day over two weeks. After 15 cycles, the evaporation rate and steam generation efficiency of the A-CG/F were still maintained at approximately $2.15 \mathrm{~kg} \mathrm{~m}^{-2} \mathrm{~h}^{-1}$ and $93.3 \%$ (Fig. 5f), respectively, which indicates that the 
sample has excellent reusability. Additionally, the salt rejection performance of A-CG/F was investigated. Various $\mathrm{NaCl}$ particles were placed on the surface of $\mathrm{A}-\mathrm{CG} / \mathrm{F}$, owing to the good hydrophilicity of A-CG/F and the macroporous structure of the cryogel, almost no salt was observed on the surface of $A-C G / F$ after being subjected to an irradiation of 1 Sun for 4 hours (Fig. S10).

As is known, the air flow in the evaporation environment is another important stimulus (N. Li et al., 2020). The effects of air flow on solar evaporation, such as the vapor temperature on the light-absorbing surface, windward surface, and leeward surface of the evaporator, were systematic investigated. Therefore, using the device shown in Fig. S11, the evaporation rate of the evaporator at different wind speeds and the vapor temperature at different evaporation positions were detected in real time. With the continuous enhancement of wind energy, the evaporation rate of A-CG/F with the wind speed of 1, 2, 3 $\mathrm{m} / \mathrm{s}$ reached $2.66,3.90$, and $5.67 \mathrm{~kg} \mathrm{~m}^{-2} \mathrm{~h}^{-1}$, respectively (Fig. 6a). The reason for this is the fact that the air flow in the evaporative environment pushes away air with higher humidity and brings in air with lower humidity such that the water in the A-CG/F can evaporate into the environment in a free manner.

Therefore, even in the absence of light, the increase of wind speed significantly increased the evaporation rate of A-CG/F (Fig. 6b). On the other hand, under windy energy the surface temperature of the A-CG/F was lower than the ambient temperature, meaning that the water steam generation can further be facilitated by the additional heat energy form the environment. This can be concluded from the facts shown in Fig. $6 \mathrm{~d}, \mathrm{e}, \mathrm{f}, \mathrm{S} 12$. When there was no wind, the A-CG/F benefited from its excellent light-heat effect. The temperature of the light-absorbing surface significantly increased and heat was conducted to the side surface. The temperature of the side surface also gradually increased even higher than the ambient temperature (Fig. 4, 6c). When the wind energy was input, the water evaporation process was accelerated accompanied with more heat removed, leading to the surface temperature of A-CG/F was lower than the ambient temperature (Fig. 6d, e, f, S12). This means that, during the evaporation process assisted by wind energy, A-CG/F cannot only receive solar energy from the sun, but can also absorb heat from the environment, which can contribute to the water steam generation. Additionally, the temperature on the windward side was significantly higher than that on the leeward side (Fig. 6d-f, S12), which is attributed to the changes in the wind speed and evaporation rate at different locations. Notably, the evaporation rate of the A-CG/F is very competitive compared with previously reported interfacial steam generators (Table S2) (Chen et al., 2020; Jiang et al., 2018; N. Li et al., 2020; X. Li et al., 2018; Mu et al., 2019; Tan, Wang, Song, Fang, \& Zhang, 2019; Y. Wang et al., 2018; Xiao et al., 2019; Yin et al., 2018; X. Zhou, Zhao, et al., 2019; Xingyi Zhou, Zhao, Guo, Zhang, \& Yu, 2018). This is an inevitable consequence of our comprehensive consideration of the lower evaporation enthalpy of water in the cryogel swelled by water, greater evaporation areas, and additional energy input.

To demonstrate the solar water purification performance of A-CG/F, solar evaporation tests were conducted on various types of raw water under the irradiation of 1 Sun, and the quality of the collected water was evaluated. Figure $7 \mathrm{a}$ shows the solar desalination ability of $\mathrm{A}-\mathrm{CG} / \mathrm{F}$ on $3.5 \mathrm{wt} \% \mathrm{NaCl}, 3 \mathrm{wt} \%$ mixed salt solution and $\mathrm{AgNO}_{3}$ solution. The initial concentration of $\mathrm{Na}^{+}, \mathrm{K}^{+}, \mathrm{Ca}^{2+}$, and $\mathrm{Mg}^{2+}$ was significantly reduced by approximately four orders, which is far less than that specified in the drinking 
water standard of the World Health Organization. Even for $\mathrm{Ag}^{+}$, which is required in drinking water, the requirements for pure water after solar desalination are fully satisfied. Notably, A-CG/F cannot only remove the ions in the brine, but can also purify various types of raw water and maintain a high steam generation rate (Fig. 7b). After the purification of wastewater rich in methylene blue and rhodamine $B$, the absorption peak disappeared, which again confirms the powerful purification ability of A-CG/F (Fig. 7c, d). These results demonstrate the potential of $A-C G / F$ for use in seawater desalination, and domestic and industrial wastewater purification.

\section{Conclusion}

In summary, the cryogel and aerogel are effectively bonded to form a novel A-CG with hierarchically porous structures. The macroporous cryogel rich in photothermal materials can effectively absorb solar energy (light absorption of $98 \%$ ), and activate the water (evaporation enthalpy of $1.66 \mathrm{~kJ} \mathrm{~g}^{-1}$ ). The ultrahydrophilic aerogel with relatively small pores endows the A-CG with self-floating properties, stronger capillary force, and reduced heat loss. The optimized A-CG/F with improved thermal management exhibited an outstanding evaporation rate of up to $2.16 \mathrm{~kg} \mathrm{~m}^{-2} \mathrm{~h}^{-1}$ under the irradiation of 1 Sun, which corresponds to a steam generation efficiency of $93.6 \%$. Moreover, in a windy environment, the A-CG/F can also effectively absorb heat from the environment. Therefore, a remarkable high evaporation rate of 5.67 $\mathrm{kg} \mathrm{m}^{-2} \mathrm{~h}^{-1}$ was achieved at the wind speed of $3 \mathrm{~m} \mathrm{~s}^{-1}$. This is an inevitable consequence of our comprehensive consideration of the lower evaporation enthalpy of water in the cryogels, greater evaporation areas, and additional energy input. The A-CGs can achieve high purification for various types of raw water. With these promising properties, these environmentally efficient and sustainable A-CGs have great potential for use in seawater desalination, and domestic and industrial wastewater purification.

\section{Declarations}

\section{Supporting Information}

Supplementary experimental methods, including equivalent evaporation enthalpy and thermal conductivity test methods, energy balance analysis during evaporation and related characterization results, including Fig. S1-S12 and Table S1-S2.

\section{Conflict of Interest}

The authors declare no competing financial interests.

\section{Acknowledgements}

The authors gratefully acknowledge the financial support of the National Natural Science Foundation of China (21664006), Natural Science Foundation of Guangxi Province (2016GXNSFAA380004), Key 
Laboratory of New Processing Technology for Nonferrous Metal \Materials, Ministry of Education/ Guangxi Key Laboratory of Optical and Electronic Materials and Devices (20KF-1 and 20AA-12), Bagui Scholar Program of Guangxi Province.

\section{References}

1. Chen, G., Li, N., He, J., Qiao, L., Li, F., Wang, S., \& Xu, X. (2020). Design of self-righting steam generators for solar-driven interfacial evaporation and self-powered water wave detection. Journal of Materials Chemistry A, 8(46), 24664-24674.

2. Crabtree, G. W., \& Lewis, N. S. (2007). Solar energy conversion. Physics Today, 60(3), 37-42.

3. Guo, Y., Zhou, X., Zhao, F., Bae, J., Rosenberger, B., \& Yu, G. (2019). Synergistic Energy Nanoconfinement and Water Activation in Hydrogels for Efficient Solar Water Desalination. ACS Nano, 13(7), 7913-7919.

4. Jiang, F., Liu, H., Li, Y., Kuang, Y., Xu, X., Chen, C., \& Hu, L. (2018). Lightweight, Mesoporous, and Highly Absorptive All-Nanofiber Aerogel for Efficient Solar Steam Generation. ACS Applied Materials \& Interfaces, 10(1), 1104-1112.

5. Li, J., Wang, X., Lin, Z., Xu, N., Li, X., Liang, J., \& Zhu, J. (2020). Over $10 \mathrm{~kg} \mathrm{~m}^{-2} \mathrm{~h}^{-1}$ Evaporation Rate Enabled by a 3D Interconnected Porous Carbon Foam. Joule, 4(4), 928-937.

6. Li, N., Qiao, L., He, J., Wang, S., Yu, L., Murto, P., \& Xu, X. (2020). Solar-Driven Interfacial Evaporation and Self-Powered Water Wave Detection Based on an All-Cellulose Monolithic Design. Advanced Functional Materials, 31(7), 2008681.

7. Li, S., He, Y., Wang, Y., Liao, D., Liu, H., Zhou, L., \& Chen, Y. (2020). Simple Hierarchical Interface Design Strategy for Accelerating Solar Evaporation. Macromolecular Materials and Engineering, 306(3), 2000640.

8. Li, S., Zhou, C., He, Y., Liu, H., Zhou, L., Yu, C., \& Wang, C. (2020). Novel Nanocellulose/Polymer Composite Aerogel as Solid-State Fluorescence Probe by Pickering Emulsion Route. Macromolecular Materials and Engineering, 305(11), 2000640.

9. Li, T., Liu, H., Zhao, X., Chen, G., Dai, J., Pastel, G., \& Hu, L. (2018). Scalable and Highly Efficient Mesoporous Wood-Based Solar Steam Generation Device: Localized Heat, Rapid Water Transport. Advanced Functional Materials, 28(16), 1707134.

10. Li, W., Li, Z., Bertelsmann, K., \& Fan, D. E. (2019). Portable Low-Pressure Solar Steaming-Collection Unisystem with Polypyrrole Origamis. Advanced Materials, 31(29), 1900720.

11. Li, X., Li, J., Lu, J., Xu, N., Chen, C., Min, X., \& Zhu, J. (2018). Enhancement of Interfacial Solar Vapor Generation by Environmental Energy. Joule, 2(7), 1331-1338.

12. Li, X., Ni, G., Cooper, T., Xu, N., Li, J., Zhou, L., \& Zhu, J. (2019). Measuring Conversion Efficiency of Solar Vapor Generation. Joule, 3(8), 1798-1803.

13. Liu, H., Huang, Z., Liu, K., Hu, X., \& Zhou, J. (2019). Interfacial Solar-to-Heat Conversion for Desalination. Advanced Energy Materials, 9(21), 1900310. 
14. Mu, P., Zhang, Z., Bai, W., He, J., Sun, H., Zhu, Z., \& Li, A. (2019). Superwetting Monolithic HollowCarbon-Nanotubes Aerogels with Hierarchically Nanoporous Structure for Efficient Solar Steam Generation. Advanced Energy Materials, 9(1), 1802158.

15. Ni, G., Li, G., Boriskina, S. V., Li, H., Yang, W., Zhang, T., \& Chen, G. (2016). Steam generation under one sun enabled by a floating structure with thermal concentration. Nature Energy, 1(9), 16126.

16. Shalkevich, N., Shalkevich, A., \& Burgi, T. (2010). Thermal Conductivity of Concentrated Colloids in Different States. The Journal of Physical Chemistry C, 114(21), 9568-9572.

17. Shu, Y., Bai, Q., Fu, G., Xiong, Q., Li, C., Ding, H., \& Uyama, H. (2020). Hierarchical porous carbons from polysaccharides carboxymethyl cellulose, bacterial cellulose, and citric acid for supercapacitor. Carbohydrate Polymers, 227, 115346.

18. Tan, M., Wang, J., Song, W., Fang, J., \& Zhang, X. (2019). Self-floating hybrid hydrogels assembled with conducting polymer hollow spheres and silica aerogel microparticles for solar steam generation. Journal of Materials Chemistry A, 7(3), 1244-1251.

19. Tao, P., Ni, G., Song, C., Shang, W., Wu, J., Zhu, J., \& Deng, T. (2018). Solar-driven interfacial evaporation. Nature Energy, 3(12), 1031-1041.

20. Wang, L., Liu, C., Wang, H., Xu, Y., Ma, S., Zhuang, Y., \& Yang, H. (2020). Three-Dimensional WoodInspired Bilayer Membrane Device Containing Microchannels for Highly Efficient Solar Steam Generation. ACS Applied Materials \& Interfaces, 12(21), 24328-24338.

21. Wang, Y., Wang, C., Song, X., Huang, M., Megarajan, S. K., Shaukat, S. F., \& Jiang, H. (2018). Improved light-harvesting and thermal management for efficient solar-driven water evaporation using 3D photothermal cones. Journal of Materials Chemistry A, 6(21), 9874-9881.

22. Wang, Z., Wu, X., He, F., Peng, S., \& Li, Y. (2021). Confinement Capillarity of Thin Coating for Boosting Solar-Driven Water Evaporation. Advanced Functional Materials, 31(22), 2011114.

23. Wu, X., Gao, T., Han, C., Xu, J., Owens, G., \& Xu, H. (2019). A photothermal reservoir for highly efficient solar steam generation without bulk water. Science Bulletin, 64(21), 1625-1633.

24. Xiao, P., Gu, J., Zhang, C., Ni, F., Liang, Y., He, J., \& Chen, T. (2019). A scalable, low-cost and robust photo-thermal fabric with tunable and programmable 2D/3D structures towards environmentally adaptable liquid/solid-medium water extraction. Nano Energy, 65, 104002.

25. Xu, Y., Ma, J., Han, Y., Zhang, J., Cui, F., Zhao, Y., \& Wang, W. (2019). Multifunctional CuO Nanowire Mesh for Highly Efficient Solar Evaporation and Water Purification. ACS Sustainable Chemistry \& Engineering, 7(5), 5476-5485.

26. Yin, X., Zhang, Y., Guo, Q., Cai, X., Xiao, J., Ding, Z., \& Yang, J. (2018). Macroporous Double-Network Hydrogel for High-Efficiency Solar Steam Generation Under 1 sun Illumination. ACS Applied Materials \& Interfaces, 10(13), 10998-11007.

27. Yu, Z.-P., Yan, K., Ullah, W., Chen, H., \& Li, C.-Z. (2020). Conjugated Polymers for Photon-to-Electron and Photon-to-Fuel Conversions. ACS Applied Polymer Materials, 3(1), 60-92.

28. Yuan, Y., Dong, C., Gu, J., Liu, Q., Xu, J., Zhou, C., \& Zhang, D. (2020). A Scalable Nickel-Cellulose Hybrid Metamaterial with Broadband Light Absorption for Efficient Solar Distillation. Advanced 
Materials, 32(17), 1907975.

29. Zhang, P., Li, J., Lv, L., Zhao, Y., \& Qu, L. (2017). Vertically Aligned Graphene Sheets Membrane for Highly Efficient Solar Thermal Generation of Clean Water. ACS Nano, 11(5), 5087-5093.

30. Zhang, P., Liao, Q., Yao, H., Huang, Y., Cheng, H., \& Qu, L. (2019). Direct solar steam generation system for clean water production. Energy Storage Materials, 18, 429-446.

31. Zhou, X., Guo, Y., Zhao, F., Shi, W., \& Yu, G. (2020). Topology-Controlled Hydration of Polymer Network in Hydrogels for Solar-Driven Wastewater Treatment. Advanced Materials, 32(52), 2007012.

32. Zhou, X., Guo, Y., Zhao, F., \& Yu, G. (2019). Hydrogels as an Emerging Material Platform for Solar Water Purification. Accounts of Chemical Research, 52(11), 3244-3253.

33. Zhou, X., Zhao, F., Guo, Y., Rosenberger, B., \& Yu, G. (2019). Architecting highly hydratable polymer networks to tune the water state for solar water purification. Science Advances, 5(6), eaaw5484.

34. Zhou, X., Zhao, F., Guo, Y., Zhang, Y., \& Yu, G. (2018). A hydrogel-based antifouling solar evaporator for highly efficient water desalination. Energy \& Environmental Science, 11(8), 1985-1992.

35. Zou, Y., Chen, X., Yang, P., Liang, G., Yang, Y., Gu, Z., \& Li, Y. (2020). Regulating the absorption spectrum of polydopamine. Science Advances, 6(36), eabb4696.

\section{Figures}



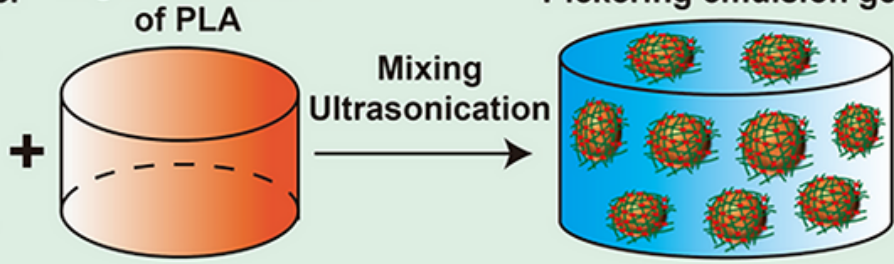
crosslinked by GA
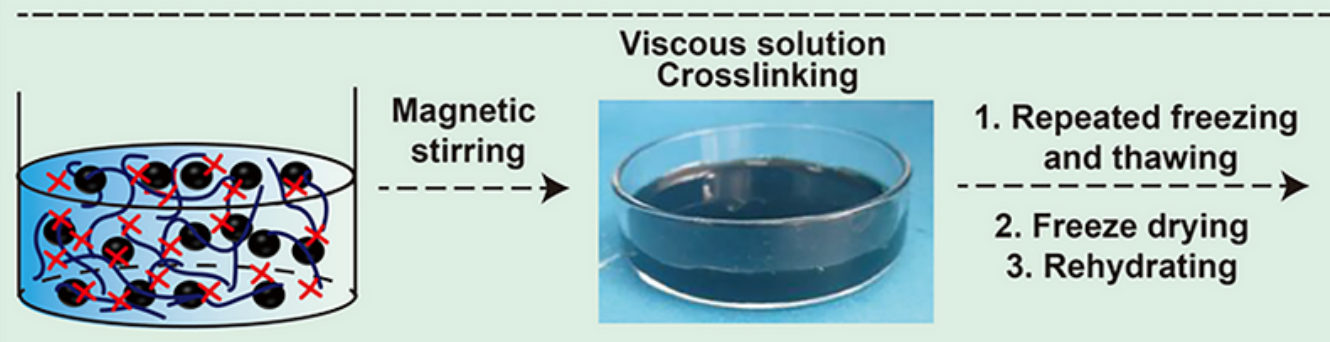

Macroporous cryogel

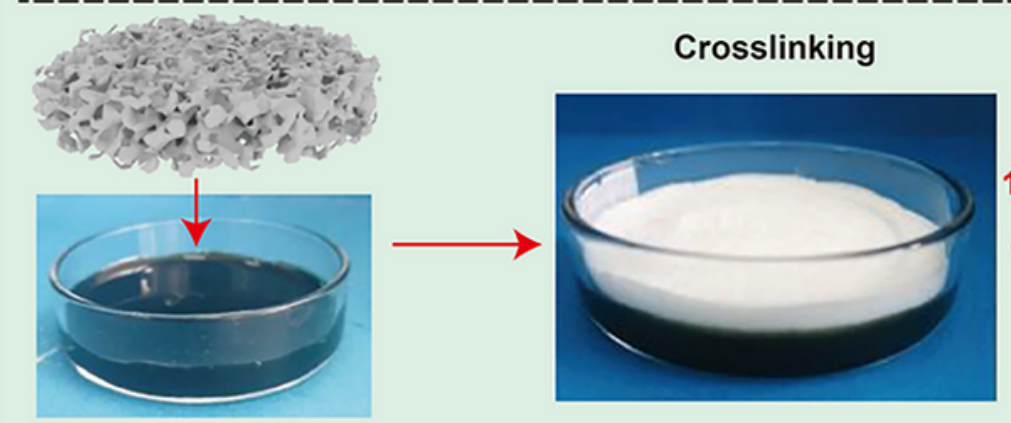

1. Repeated freezing

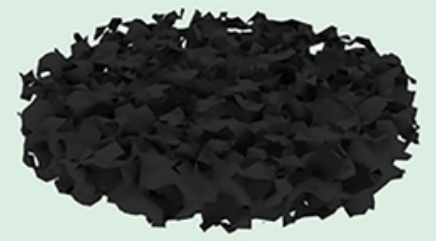

Freeze drying
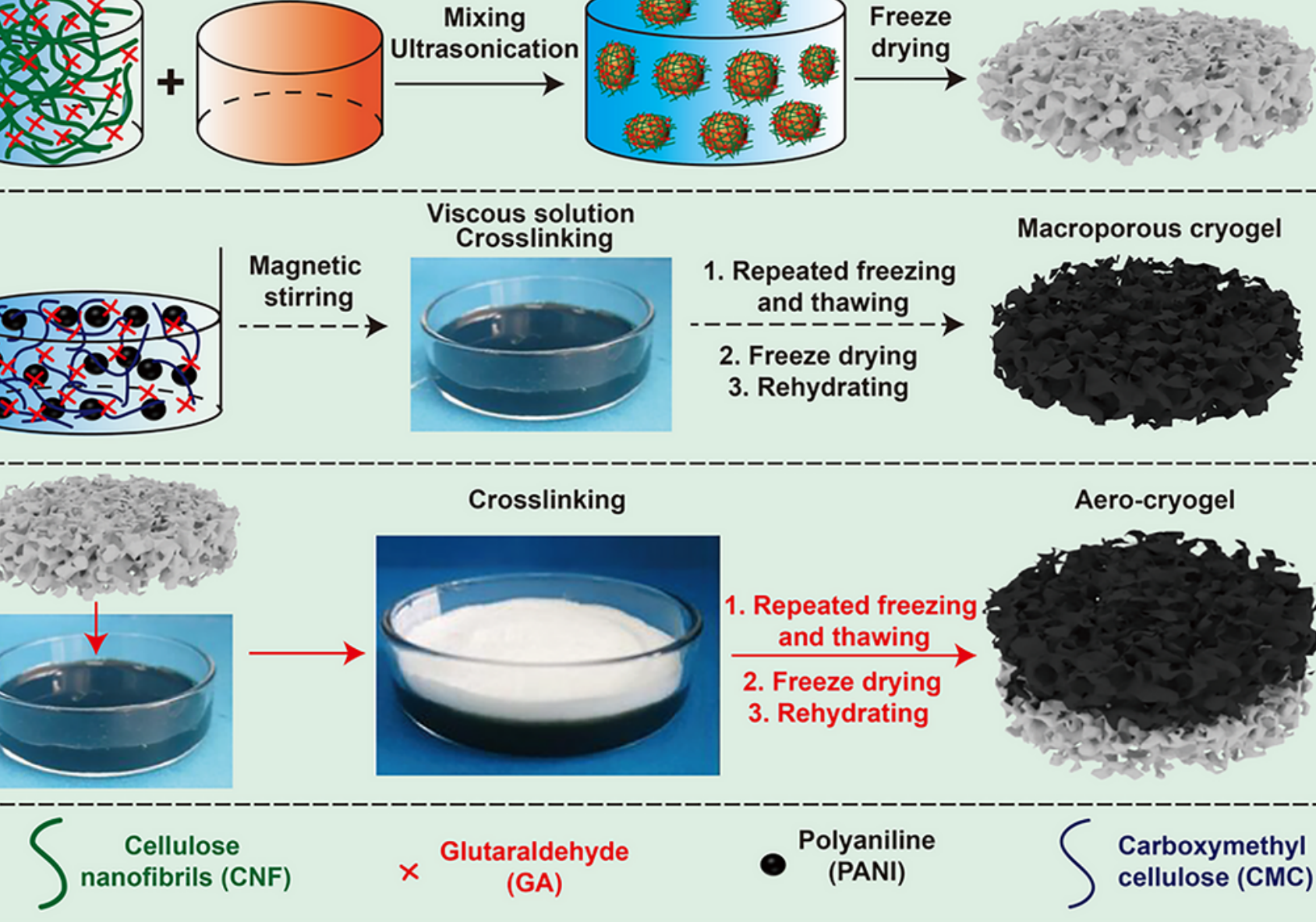

$\times$ Glutaraldehyde

(GA)
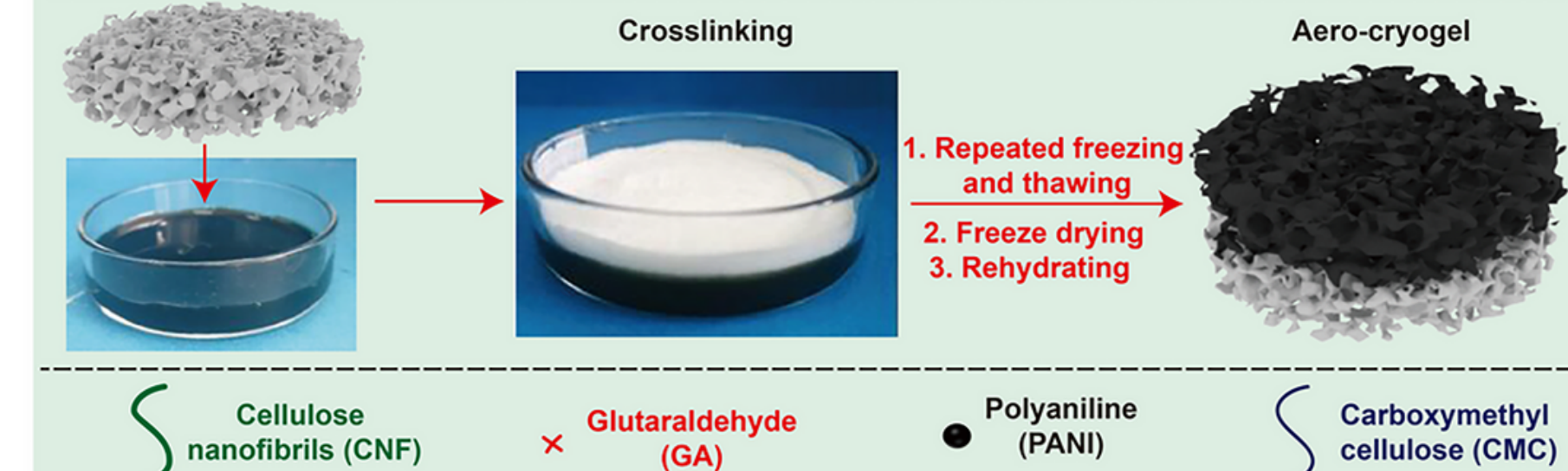

b

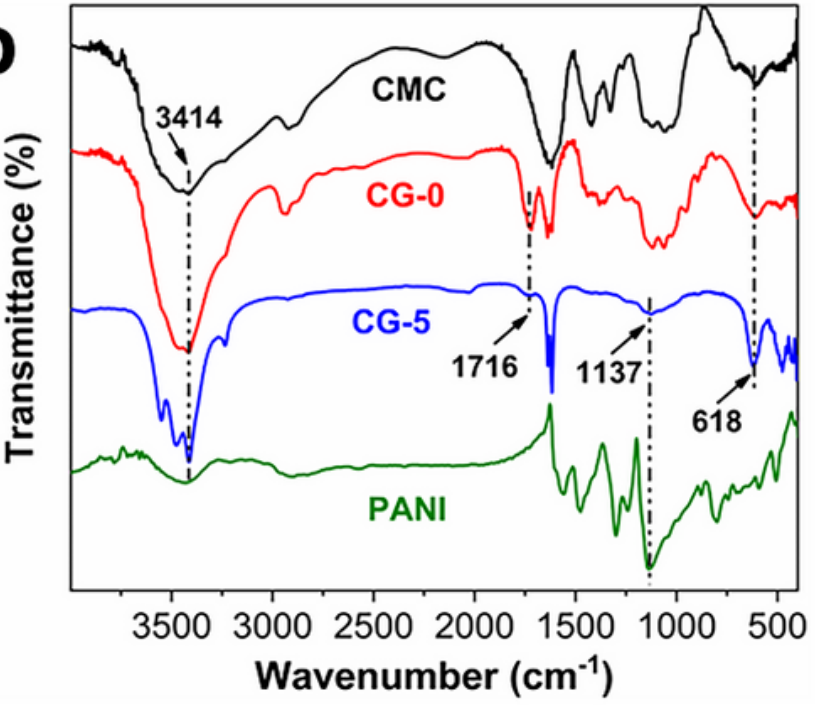

C

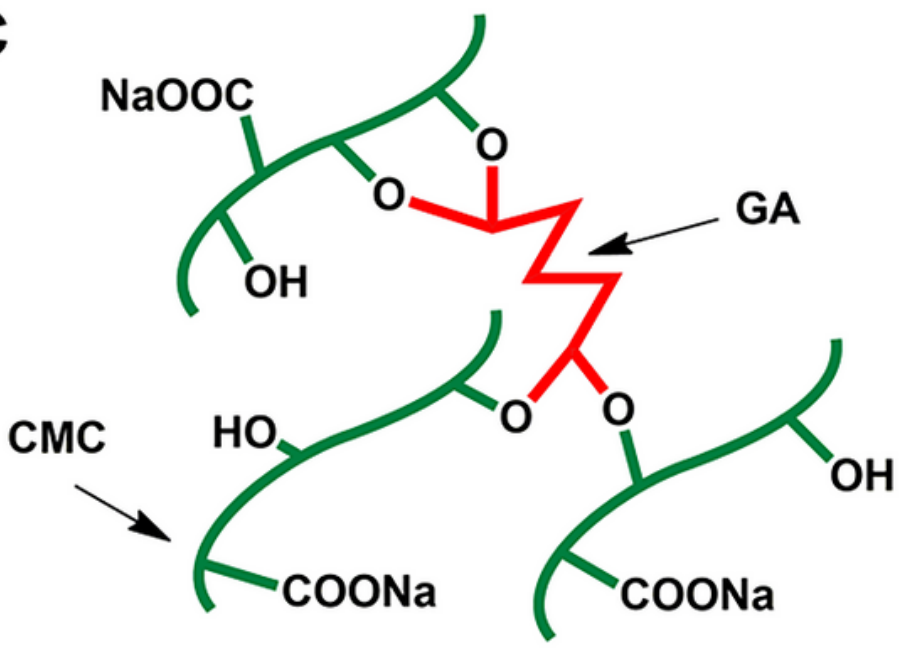

Figure 1

The preparation of aero-crygels. (a) Schematic illustration of the fabrication process of aerogel, cryogel and aero-cryogel monoliths, (b) FTIR spectra of PANI, CG-5, CG-0 and CMC, (c) carboxymethyl cellulose (CMC) polymer cross-linked by glutaraldehyde (GA) 

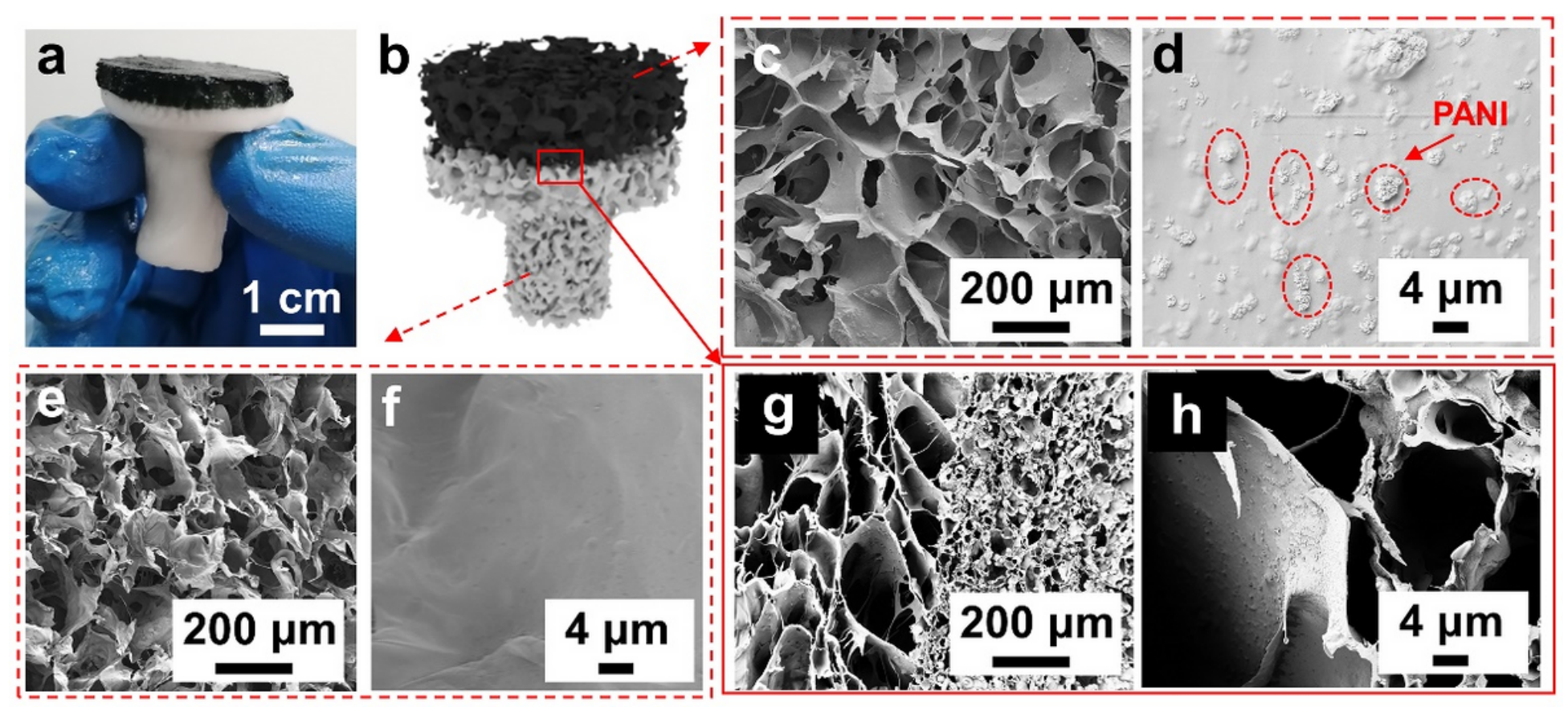

Figure 2

The macroporous structure of aero-cryogels. (a) Digital photo and (b) schematic illustration of an integrated A-CG-5 (c, d) the porous aerogel phase of CG-5, (e, f) the porous aerogel phase of CG-5 and ( $g$, h) the interface of cryogel and aerogel phases 

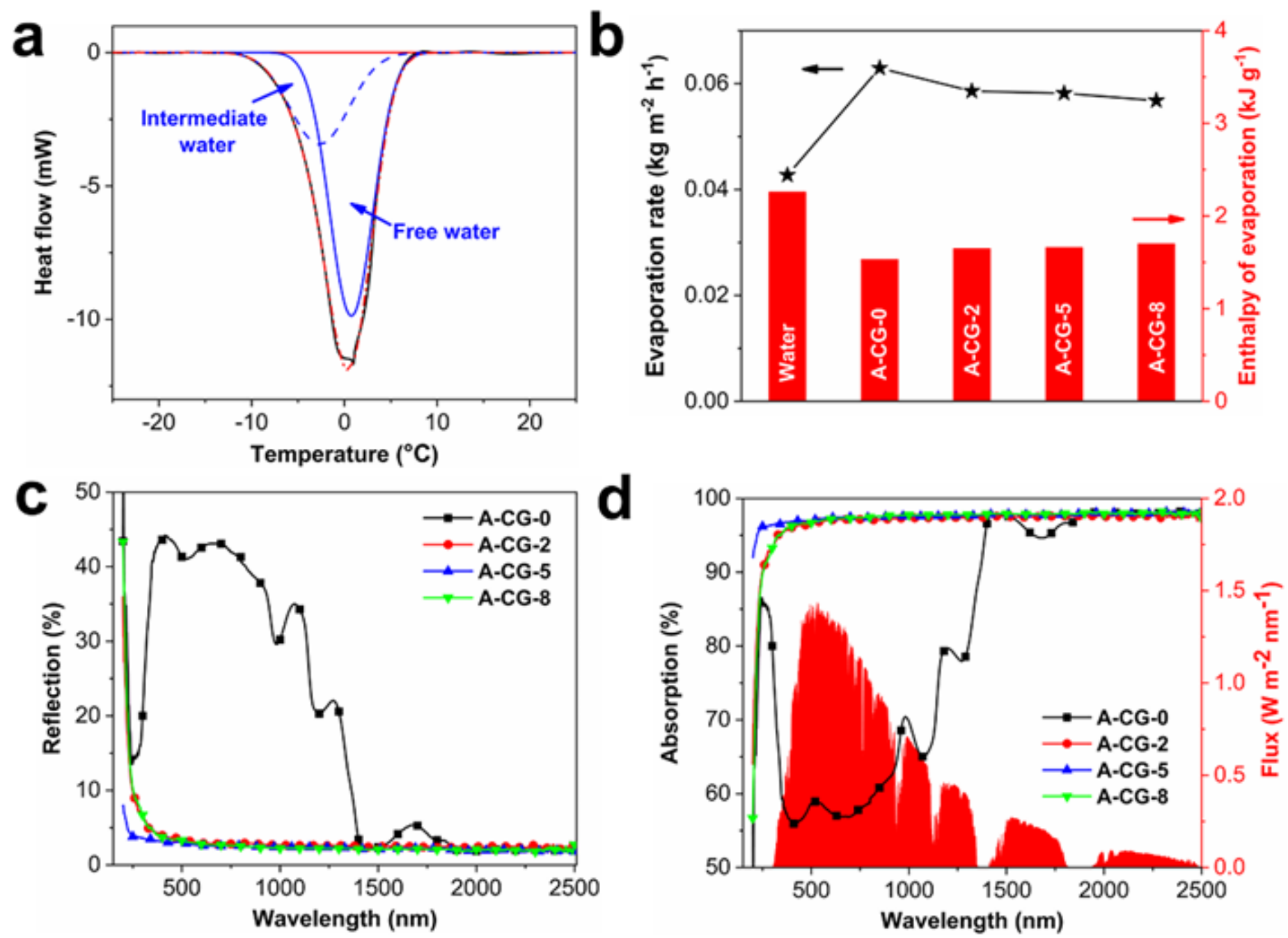

Figure 3

The water evaporation in aero-cryogels. (a) Differential scanning calorimetry curves of CG-5, (b) the equivalent water vaporization enthalpy and water evaporation rate of A-CGs in dark, UV-vis-NIR (c) reflectance and (d) absorption spectra of A-CGs 


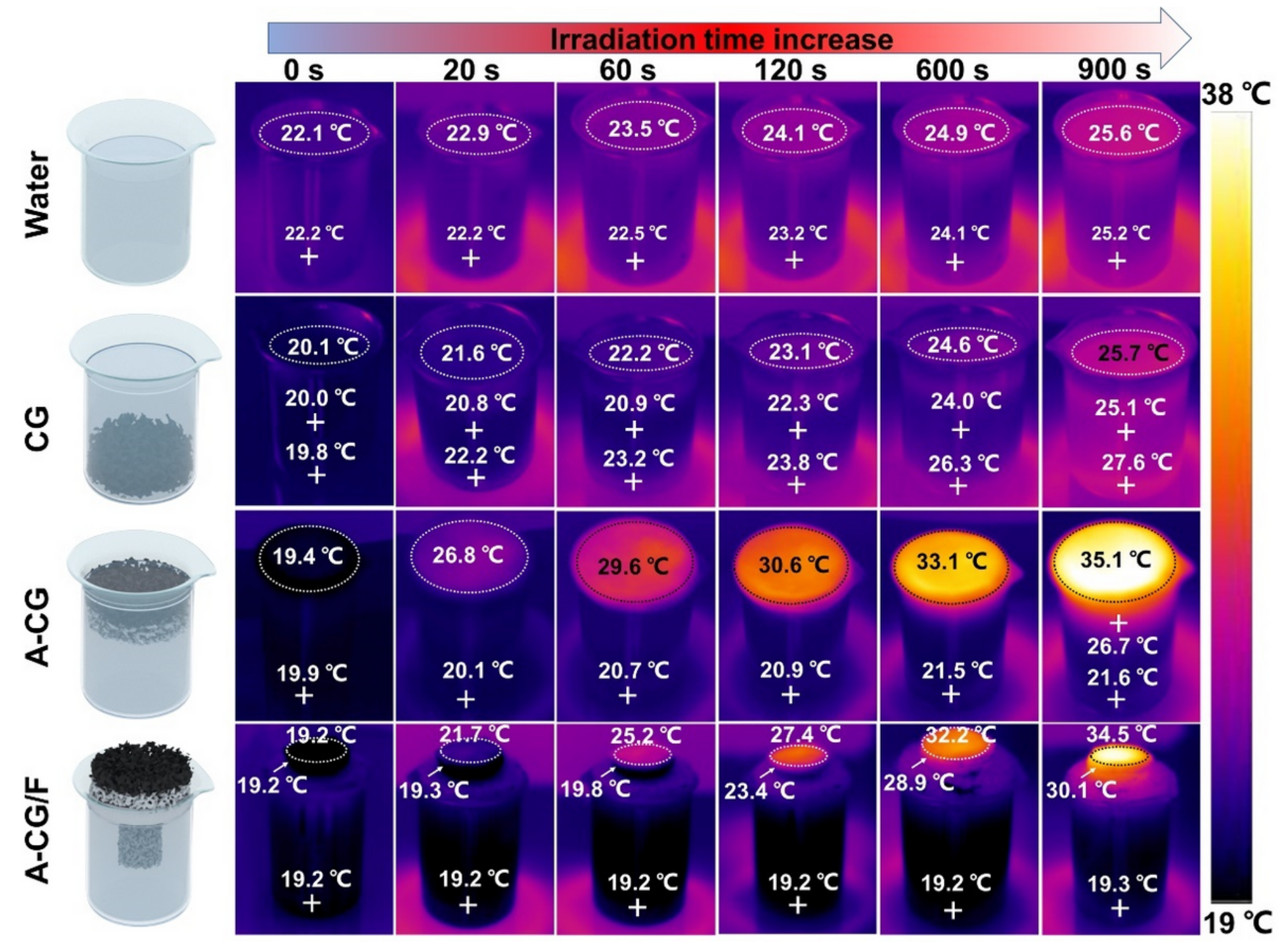

Figure 4

The infrared thermal images of water, CG, A-CG, A-CG/F under irradiation of 1 Sun for $900 \mathrm{~s}$ 

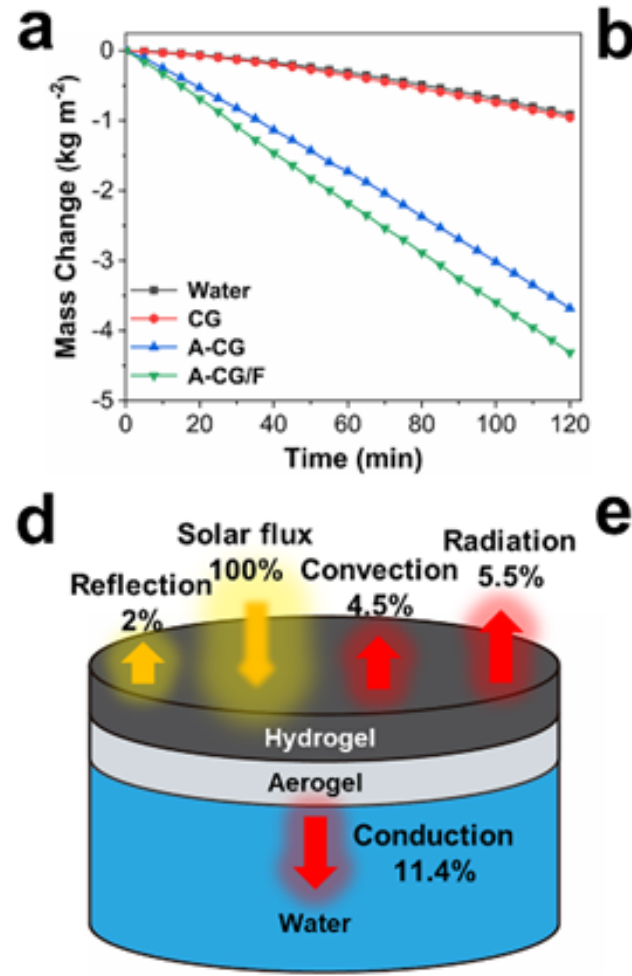

e
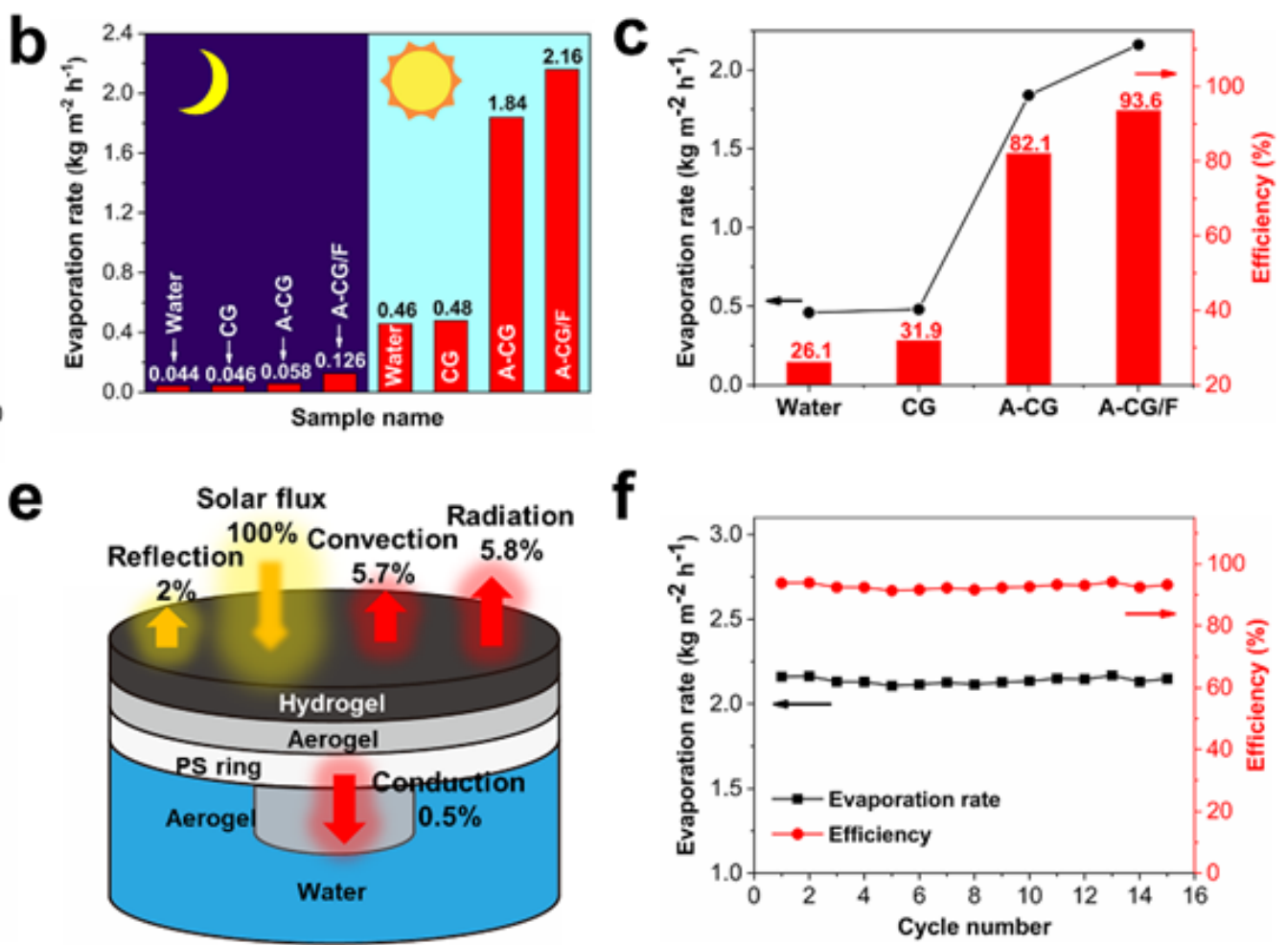

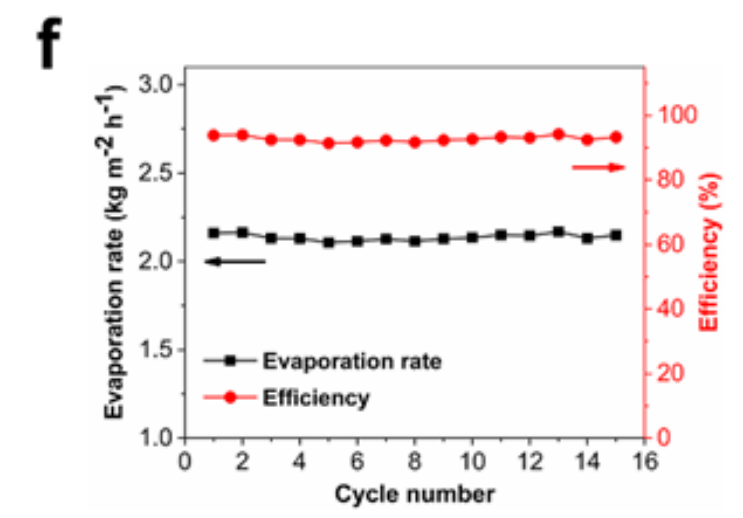

Figure 5

Water evaporation and steam efficiency of aero-cryogel solar steam devices. (a) Evaporation mass loss for different evaporation devices under irradiation of 1 Sun for two hours, (b) evaporation rate of bulk water and SVGs in dark condition andirradiation condition, (c) evaporation rate and efficiency of bulk water and SVGs under irradiation of 1 Sun., and a heat loss diagram of (d) A-CG and (e) A-CG/F, (f) evaporation rates and steam generation efficiency of $A-C G / F$ with repeated operations 

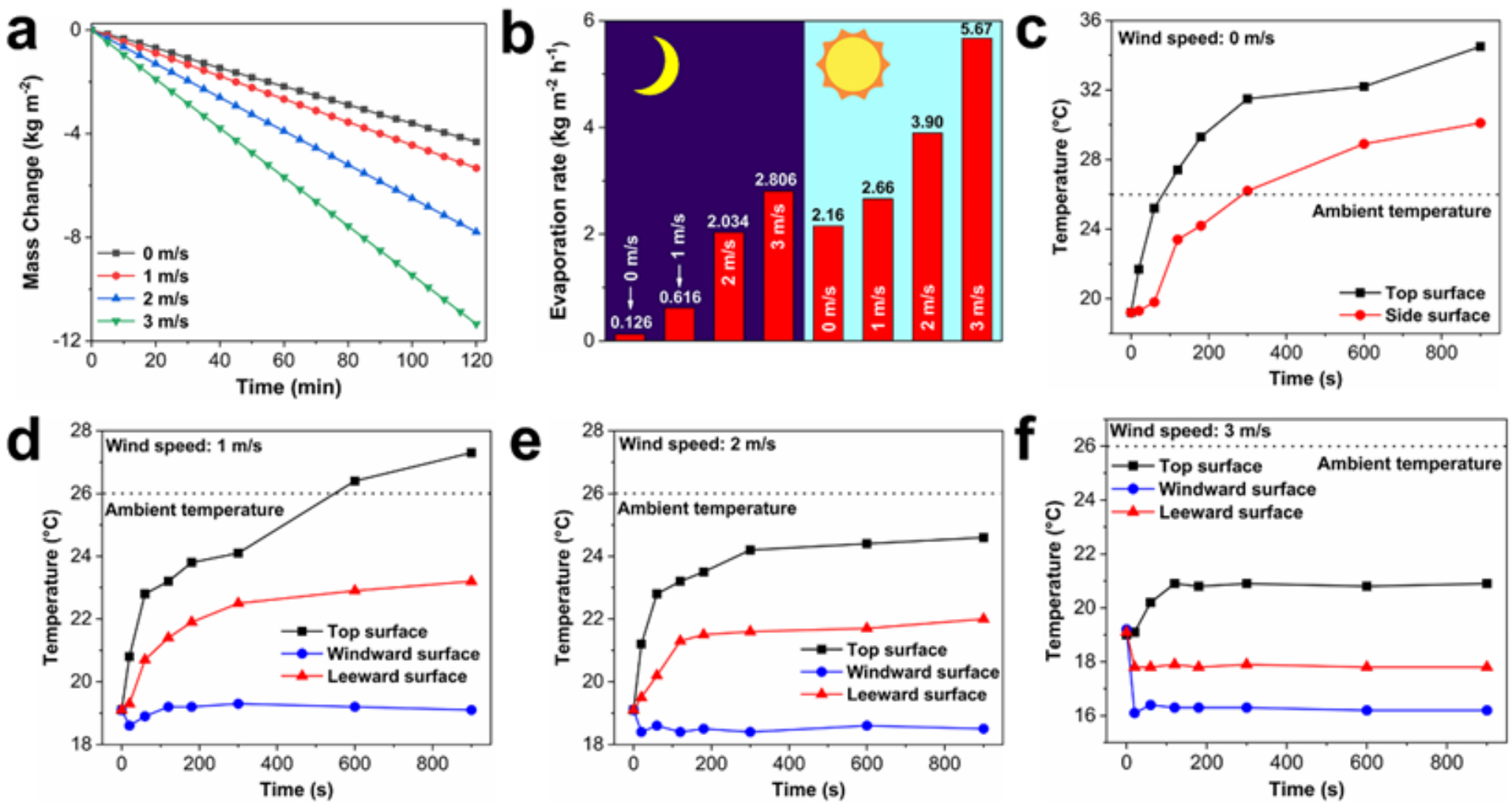

Figure 6

Wind energy enhanced water evaporation and steam efficiency of aero-cryogel solar steam devices. (a) Evaporation mass loss for A-CG/F under irradiation of 1 Sun at different wind speeds for two hours, (b) evaporation rate of $A-C G / F$ in dark condition and irradiation condition at different wind speeds, (c-f) the surface temperature (determined by IR camera) of A-CG/F under irradiation of 1 Sun at different wind speeds 

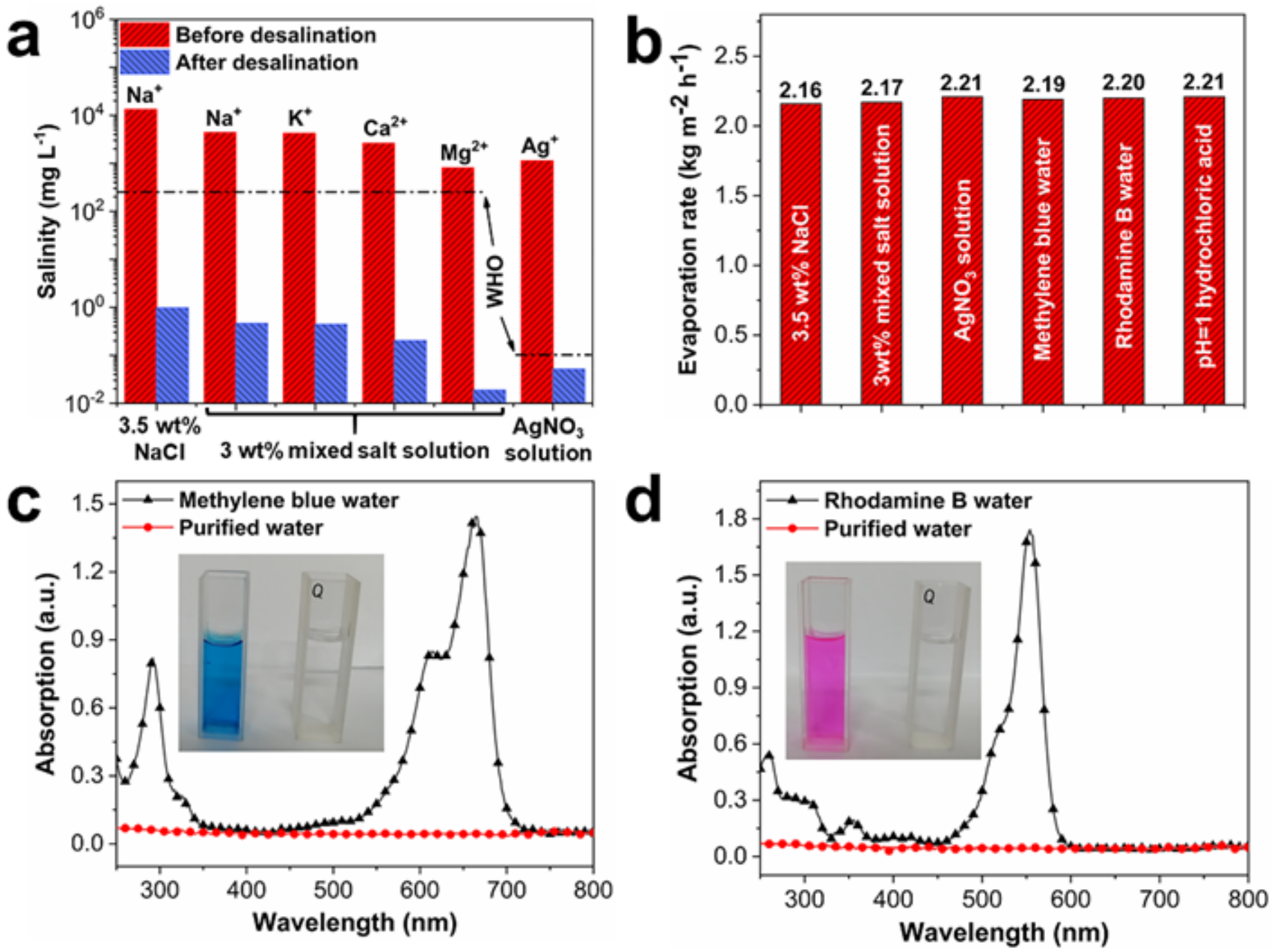

Figure 7

Solar water purification performance of aero-cryogel device. (a) Ion concentration change before and after desalination using $\mathrm{A}-\mathrm{CG} / \mathrm{F}$, (b) evaporation rates of different waters under an irradiation of 1 Sun, the UV-vis spectra of (c) methylene blue water and (d) rhodamine B water before and after solar thermal purification with A-CG/F; inset: color change of wastewater before (left) and after (right) purification, respectively

\section{Supplementary Files}

This is a list of supplementary files associated with this preprint. Click to download.

- Graphicalabstract.png

- Supplementalfiles.docx 